\title{
Research on Blood Supply Chain Simulation Based on Cost and Shortage Rate
}

\author{
Wenjuan Fan $^{1, a}$ \\ ${ }^{1}$ Beijing Jiaotong University, Beijing, China \\ a16120573@bjtu.edu.cn
}

Keywords: blood supply simulation; cost; shortage rate

\begin{abstract}
Based on the MBB case in India, this paper takes the cost and the shortage rate as output variables, considers five events including demand events, collection events, storage events, shortage events, and overdue events in the blood supply chain, and establishes a blood supply chain simulation model. The total cost and shortage rate under normal circumstances, expanding blood collection station scale, and increasing the number of organized blood collection activities provide decision-making suggestions for decision makers. The simulation results of the study examples show that expanding the scale of blood collection stations and increasing the size of blood collection stations can make the total cost decreased by $68 \%$, the shortage rate decreased by an average of $26 \%$, the increase in the number of blood collection activities reduced the total cost of the blood supply chain by $61 \%$, and the shortage rate decreased by $33 \%$, which verified the feasibility and effectiveness of the simulation model. Research on other blood centers also has some value of reference.
\end{abstract}

\section{Introduction}

With the continuous improvement of medical standards, the demand for blood for clinical use continues to rise. Blood is a special resource. There is evidence that transfusions stored for more than 14 days can lead to sepsis and death in trauma patients ${ }^{[1]}$ The blood supply chain is fundamentally different from other supply chains. The related blood supply chains in foreign countries are mainly focused on the blood supply chain network design, emergency blood dispatch optimization, blood center inventory management, and blood supply. There are few researches on the blood supply chain in China, such as location selection of logistics nodes, blood transport problems, and blood supply chain simulation ${ }^{[2-3]}$, etc. Emergency blood transport and scheduling, demand forecasting, etc. Since the donor's blood supply is random, the hospital's demand for blood and its products is also random, and the blood and its products are easily degraded in a short time. Therefore, the blood supply chain is an extremely complex system, and due to the system When the complexity is difficult to obtain the optimal solution, it is a more feasible solution to study the blood supply chain by establishing the simulation model to evaluate the performance of the system or optimize the related parameters of the system. Simulation research mainly aims to improve the efficiency of blood supply chain ${ }^{[2-3]}$ or optimize inventory management ${ }^{[4-6]}$ to reduce the shortage rate. For blood centers, reducing the shortage rate is important, but as a public welfare organization, It is also very important to reduce the shortage rate while controlling costs. This article uses a simulation method to establish a simulation model that meets the blood supply chain's actual flow, using the case of IVEY's authorized use, the Indian Model Blood Bank (MBB), to reduce blood flow. The supply chain cost and shortage rate provide the basis for decision-making, while providing reference for the study of other blood centers, the results of the study are realistic Righteousness.

\section{MBB Blood supply chain}

MBB is the largest blood bank in Indore City and was established in 1955. It is a non-profit blood bank within the government-owned Maharaja Yeshwant Rao Hospital (commonly known as "MY Hospital"). The blood bank is operated jointly by the Indian Hospital and Mahatma Gandhi Memorial 
Medical College. The State Government and the National AIDS Control Organization provide funds for the operation of the National Blood Bank. The MBB team consists of 35 responsible persons. The blood bank operates 24 hours a day, seven days a week, and provides high quality blood products to various hospitals in the city.

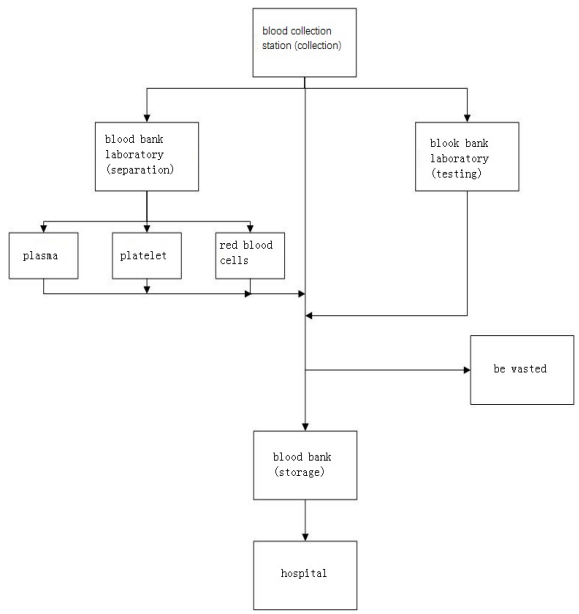

Fig. 1. Blood supply chain in MBB

\subsection{Problem description and solution}

With the increase in demand for blood from hospitals, despite the MBB's continuous efforts to increase blood collection, the number of blood shortages continues to increase. During the shortage of blood supply, the blood banks of each hospital will usually order 2-3 times than the amount of blood actual needed. The supply chain is more unstable and it also exacerbates blood shortages and blood wastage. From the actual point of view, put forward two kinds of feasible plans. First, increase the amount of blood supply by expanding the size of the existing blood collection stations and increasing publicity. Second, increase the amount of blood supply by increasing the number of blood collection activities currently held. While the scale or increase in the number of blood collection campaigns may increase costs while failing to alleviate the current status of the blood supply chain and its instability, MBB's decision makers cannot decide which plan should be adopted. This article through the simulation to determine the effectiveness of the two programs to reduce costs and shortage rate, select the optimal program. Because red blood cells are used most frequently in medical treatment, this article focuses on red blood cells.

\subsection{Symbols and basic assumptions}

The basic symbolic representations and assumptions in this article are shown in Table 1: 
Table 1. The symbols used in simulation model

\begin{tabular}{|c|c|}
\hline Symbols & Definition \\
\hline $\mathrm{k}$ & equal to $1,2,3, \ldots, 8$, representing 8 types blood types $\mathrm{A}+, \mathrm{A}-, \mathrm{B}+, \mathrm{B}-, \mathrm{O}+, \mathrm{O}-, \mathrm{AB}+$, and $\mathrm{AB}-$, respectively. \\
\hline$D_{k}^{T}$ & denotes the predicted demand for red blood cells of the $\mathrm{k}$ blood type on day $\mathrm{t}$ \\
\hline$G_{k}^{t}$ & indicates the predicted collection amount of red blood cells for the $\mathrm{k}$ blood type on day $\mathrm{t}$ \\
\hline$\alpha$ & red cell abandonment rate \\
\hline$l_{k}^{t}$ & represents red blood cell stocks of $\mathrm{k}$ blood type on day $\mathrm{t}$ \\
\hline$S_{E^{2}}^{t}$ & indicates the shortage number of red blood cells for the $\mathrm{k}$ blood type on day $\mathrm{t}$ \\
\hline$\lambda$ & indicates the unit's blood shortage costs \\
\hline$C_{r}$ & indicates the total cost of blood out of stock \\
\hline$D_{k}^{t}$ & represents the number of expired red blood cells in the blood type $\mathrm{k}$ on day $\mathrm{t}$ \\
\hline$Y$ & indicates the unit's blood expiration cost \\
\hline$C_{n}$ & indicates the total cost of expired blood \\
\hline $\mathrm{f}$ & indicates the number of blood collected on a regular scale \\
\hline $\mathrm{n}$ & increases the number of normal blood collections $\mathrm{n}$ times \\
\hline $\mathrm{b}$ & indicates the number of large blood collection activities organized \\
\hline$\theta$ & percentage of blood volume increased when large blood collection activities are held \\
\hline$c_{1}$ & $\begin{array}{c}\text { indicates the cost of organizing a general-scale blood collection campaign, including labor costs, } \\
\text { equipment costs, etc. }\end{array}$ \\
\hline $\mathrm{C}_{2}$ & $\begin{array}{l}\text { indicates the cost of organizing a large blood collection campaign, including advertising costs, labor costs, } \\
\text { equipment costs, etc. }\end{array}$ \\
\hline $\mathrm{c}_{3}$ & indicates unit blood inventory cost \\
\hline$C_{l}$ & indicates the total cost of blood inventory \\
\hline $\mathrm{C}$ & indicates the total cost of the blood supply chain \\
\hline$\beta$ & indicates the shortage rate of the blood supply chain \\
\hline
\end{tabular}

Before making the simulation model, make the following assumptions:

(1) Suppose that $2 \%$ of the red blood cells are discarded due to disqualification after blood collection, $"-2 \%$;

(2) In order to ensure blood supply, the appropriate amount of blood expired is acceptable, and the consequences of blood shortage are more serious, so it is assumed that $\lambda>>\gamma$, that is, the loss of blood shortage is far greater than the loss of blood;

(3) Only red blood cells is considered after blood is separated;

(4) Assuming normal blood transport and storage temperatures, red blood cells can be stored in the blood warehouse for 35 days;

(5) Considering the blood supply under normal circumstances, the emergency blood supply is not considered in this paper.

\section{Building a blood supply chain simulation model}

According to the characteristics of the blood supply chain, the main events in the model are identified as blood demand events blood collection event, blood storage event, blood shortage event, and blood expiration event.

(1) Demand event. The time and quantity of demand events are completely random. The model organizes historical blood demand data, obtains the corresponding demand probability, and generates a random demand $D_{\bar{K}}^{\mathrm{E}}$ corresponding to the corresponding demand probability;

(2) Collect event. The model firstly collects historical data on the number of blood collection activities in the blood center. According to historical data on the number of blood collection times in the blood center, calculates the probability of the corresponding collection times, and collects blood according to the organization. According to the probability of the number of organizing blood collection to produce the random number $\mathrm{f}$, and then to determine whether the organization of blood 
collection stations on $t$ day, the organization of blood collection is recorded as " 1 ", because the number of no-blood donors come every day is random, so the model based on the distribution of historical blood collection data, generate random collections $G_{k}^{t}$, do not organize the collection of blood as " 0 ", there is no corresponding blood collection event, $G_{L}^{E}=0$;

(3) Store event. When receiving the demand order from the hospital, the blood warehouse needs to determine whether the inventory can meet the demand. If the inventory can't meet the order, then the shortage events will happen, the inventory level is updated to $I_{k}^{\Sigma}=0$, if it can meet the demand, then update the inventory, $\mathrm{k}$ blood type red blood cells on the $\mathrm{t}$ day inventory level and inventory total cost, shown are as formula (1)-(2);

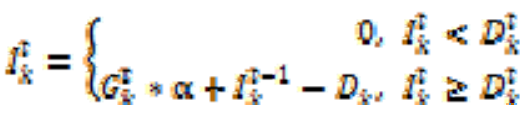

$$
\begin{aligned}
& c_{\gamma}=\Sigma_{i=1}^{\tau} \Sigma_{k=1}^{v} c_{3} * I_{k}^{\mathrm{E}}
\end{aligned}
$$

(4) Shortage event. The shortage of the number of red blood cells of the k blood type on day $t$ and the shortage cost, shown are as formula (3)-(4);

$$
\begin{aligned}
& S_{K}^{T}=D_{K}^{\tau}-I_{K}^{T} \\
& C_{S}=\Sigma_{\mathrm{i}=1}^{\tau} \sum_{i=1}^{S} \lambda * S_{\mathrm{L}}^{E}
\end{aligned}
$$

(5) Expiration event. Expiration event means that the blood storage time stored in the warehouse exceeds the red blood cell shelf life date $\left(2^{\circ} \mathrm{C} \sim 6^{\circ} \mathrm{C}\right.$, shelf life is 35 days $)$, and at the same time there is a loss of blood overdue. The number of expired red blood cells and the cost of expiration of $\mathrm{k}$ blood types on the $t$ th day, shown are as formula (5)-(6);

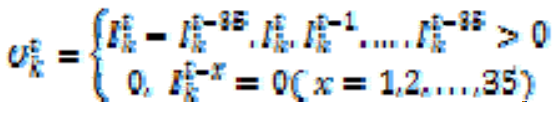

$$
\begin{aligned}
& C_{0}=\sum \sum_{i=1}^{T} \sum_{k=1} \gamma * \theta_{k}^{E}
\end{aligned}
$$

This article mainly compares the total cost of the blood supply chain $\mathrm{C}$ and the shortage rate $\beta$ under various scenarios, shown are as formula (7)-(8):

$$
\begin{aligned}
& \mathrm{C}=\mathrm{c}_{1} * f+\mathrm{c}_{2} * b+C_{3}+C_{0}+C_{0}
\end{aligned}
$$

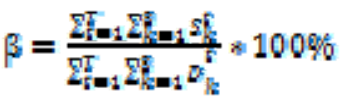

\section{Simulation case}

\subsection{Case status}

From the data released by MBB from 2011 to 2015 (shown as Fig2), there are often mismatches between supply and demand in blood centers. According to the introduction of MBB, the actual situation is worse than the published data.

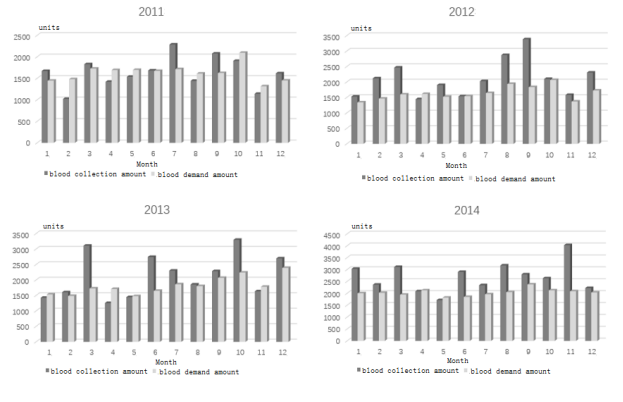


Fig. 2. MBB blood collection and demand amount from 2011 to 2014

\subsection{Data collection and analysis}

(1) Requirements data consolidation. As shown in Figure 3, the horizontal axis of each chart is the demand or demand interval (units/day), and the vertical axis shows the demand frequency. The demand process is based on historical data, and statistics the demand frequency of red blood cells. Assuming that the demand frequency is approximately equal to the demand probability of various types of red blood cells in the simulation process, the red blood cells demand random number (including $\mathrm{O}^{+}, \mathrm{O}_{-}, \mathrm{A}^{+}, \mathrm{A}_{-}, \mathrm{B}+, \mathrm{B}-, \mathrm{AB}^{+}, \mathrm{AB}_{-}$, and other eight components) are generated by using the vlookup and Rand functions.

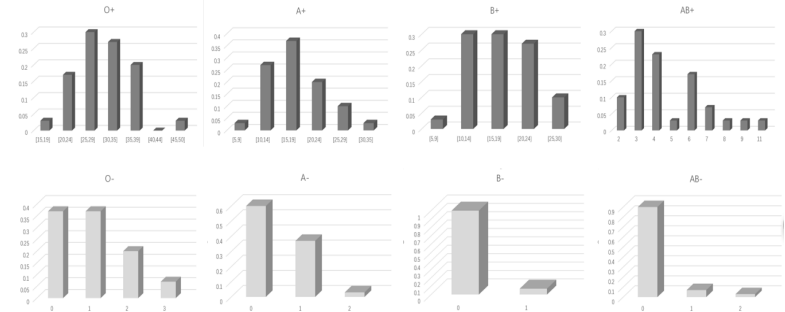

Fig. 3. Demand data for various blood red blood cells from 2011 to 2014

(2) collect data collection. The collection process includes whether to organize the blood collection station, the amount of blood collected, the amount of blood available, and the supply of red blood cells. The simulation process considers one blood collection station and multiple demand hospitals. According to the historical data of blood collection quantity, the frequency of blood collection quantity is counted. Assume that the collection quantity frequency (Fig. 4) is approximately equal to the collection probability of various types of red blood cells in the simulation process. The amount of blood collected during blood collection was calculated according to the proportion of red blood cells in the blood bank of each blood group (Table 1).

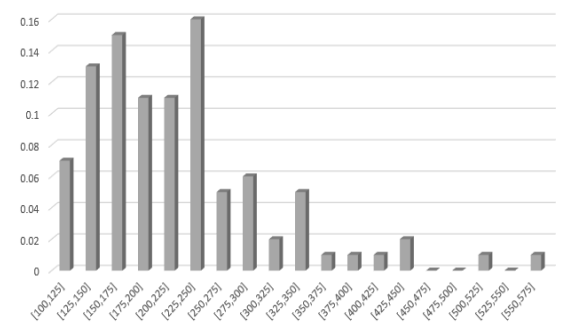

Fig. 4. Frequency chart of blood collection from 2011 to 2014

Table 2. Ratio of blood group red blood cells in blood bank

\begin{tabular}{lllllllll}
\hline Red blood group & $\mathrm{O}+$ & $\mathrm{O}-$ & $\mathrm{A}+$ & $\mathrm{A}-$ & $\mathrm{B}+$ & $\mathrm{B}-$ & $\mathrm{AB}+$ & $\mathrm{AB}-$ \\
\hline Ratio & $39 \%$ & $1 \%$ & $27 \%$ & $0.5 \%$ & $25 \%$ & $0.4 \%$ & $7 \%$ & $0.1 \%$ \\
\hline
\end{tabular}

\subsection{Simulation results}

The simulation time was set to 92 days. The software Excel and Crystal ball were used to simulate the normal state (ie, without any change), expand the existing blood collection station, and increase the number of blood collection activities. The first scenario was Expanding the scale of existing blood collection stations. The second option is to increase the number of blood collection activities. The simulation results are as follows:

(1) Normal condition

Let input variables $c_{1}=10, C_{s}=1000, C_{0}=40$. After run 1000 times, the frequency view of normal supply chain as shown in Figure 5, the simulation model output shows that the average value of the total cost of the normal case is $\mathrm{C}=2895577.94$, and the average value of the stock-out rate is $\beta=36 \%$. 


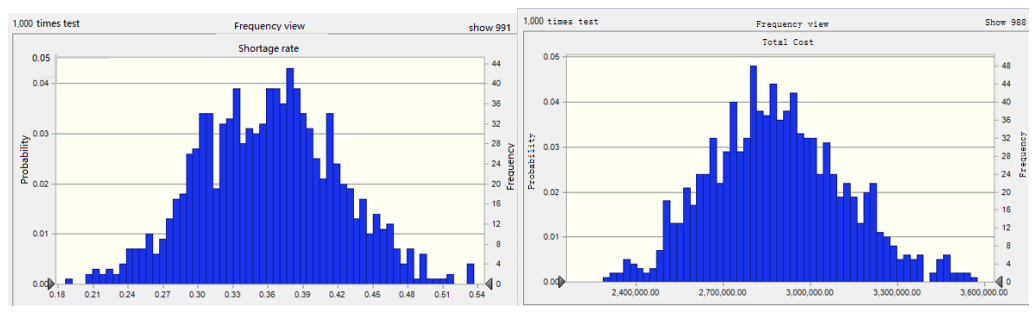

Fig. 5. Total cost and shortage rate of normal condition blood supply chain

\section{(2) Option 1}

Let the input variables $\mathrm{c}_{1}=10, \mathrm{c}_{2}=20, \theta=10 \%, C_{s}=1000, C_{0}=40$, use the IF function to determine whether the blood collection activities are organized on the $t$ day, if the cell is " 1 ", that is, organize blood collection activities. RandBetween $(0,1)$ determines whether to organize large blood collection activities, "1" indicates that the organization organizes large-scale blood collection activities, "0" indicates that large-scale blood collection activities are not organized, and the number b of large-scale organization's blood collection activities is the sum of the columns, which can be changed according to actual conditions. After run 1000 times, the frequency view of option 1 as shown in Fig. 6, the output of the simulation model of scheme 1 shows that the average value of the total cost is $C=915038.98$ and the average value of the shortage rate is $\beta=10 \%$.

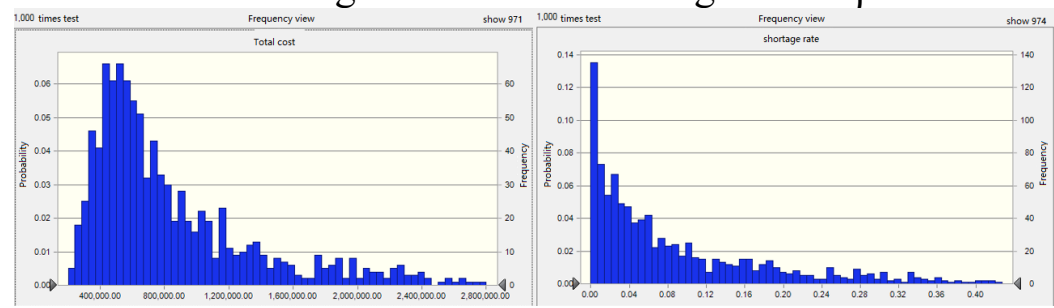

Fig. 6. Total cost and shortage rate of option 1 blood supply chain

(3) Option 2

Let input variable $\mathrm{c}_{1}=10, \mathrm{n}=10, c_{s}=1000, c_{Q}=40$. after run 1000 times, the frequency view of option 2 as shown in Fig. 7, the simulation result of the second scheme shows that the average value of the total cost is $C=1116195.85$, and the average value of the stockout rate is $\beta=3 \%$.

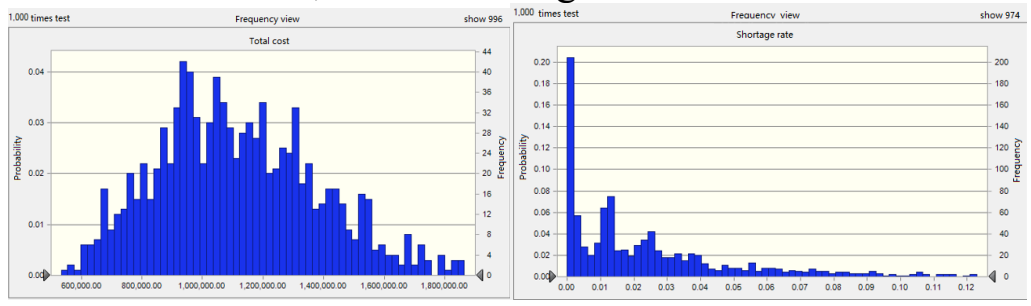

Fig. 7. Total cost and shortage rate of option 2 blood supply chain

As shown in Figure 8, the simulation results show that, compared with the normal situation, adopting plan 1 to expand the scale of the blood collection station can reduce the total cost of the blood supply chain by $68 \%$, and the shortage rate decreases by an average of $26 \%$. Increasing the number of blood collection activities can reduce the total cost of the blood supply chain by $61 \%$, and the shortage rate decreases by an average of $33 \%$. From the simulation results of the calculations, compared with the normal situation, the total cost of the blood supply chain was significantly reduced when the plan was adopted, and the rate of shortage of the plan was significantly reduced. Both plans have improved the blood supply chain. But for the blood center, the shortage of blood means that patients in the hospital cannot get the timely treatment, and it is very likely to cause serious consequences that cannot save lives. It is more important for blood centers to reduce the shortage rate, in the case study, the decision maker can choose option 2, that is, increase the number of organized blood collection activities to optimize the MBB blood supply. 


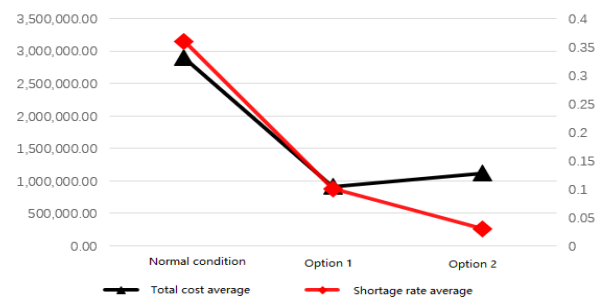

Fig. 8. Comparison of simulation optimization results

\section{Conclusion}

The blood supply chain simulation model established in this paper can be used not only in the MBB blood supply chain, but also in the blood supply chain of other blood centers. The simulation results can provide decision support for decision makers. Based on the research results of the relevant literature, the paper conducts research on the problem of high cost of blood centers and low shortage rates. Firstly, This paper proposes the statistics of the demand and the amount of acquisition in historical data according to the features of blood demand and the time and quantity of the blood collection process are completely random. Occurrence frequency, frequency instead of probability, according to the corresponding probability of generating random numbers of blood demand and blood collection random number method to predict blood demand and blood volume. Then, this paper establishes a simulation optimization model including blood demand events, blood collection events, blood storage events, blood shortage events, and general blood sampling of blood expiration events. Finally, by combining the actual historical data and operational mechanism of MBB, it is proved that the model can simulate the actual operation of the blood center and provide a basis for the blood center to reduce the supply chain cost and the shortage rate. Using the simulation model established in this paper, the cost of organizing a general-scale blood collection campaign $c_{1}$, the cost of organizing a large-scale blood collection campaign $c_{2}$, the percentage of blood collection volume $\theta$ at a large blood collection station compared to a common blood collection station, the increase in the number of general-scale blood collection activities $\mathrm{n}$, and the unit blood deficiency When parameters such as the cost of goods $\bar{C}_{s}$ and unit blood expiration cost $C_{0}$ change, the input variable data can be changed and the model can be run again to determine the pros and cons of the various scenarios in the new situation. This provides a long-term effective scientific method for blood supply management. This article simplifies the actual situation of the blood supply chain during the research process. The following studies can further study the transport path and inventory management strategies that are not considered in this paper and improve the model.

\section{References}

[1] Seyed Mojib Zahraee,Jafri Mohd Rohani,Alireza Firouzi,Ataollah Shahpanah. Efficiency Improvement of Blood Supply Chain System Using Taguchi Method and Dynamic Simulation[J]. Procedia Manufacturing,2015,2.

[2] HU Benyong,CHEN Xu,Optimization of blood supply chain with option contracts under supply and demand uncertainty[J].Systems Engineering-Theory \& Practice, 2016,36(12):3133-3141.

[3] WANG Ke-ming,MA Zu-jun,ZHOU Yu-feng,A Two-phase Decision-making Approach for Emergency Blood Transferring Problem in Public Emergencies[J].Journal of Transportation Systems Engineering and Information Technology, 2013,13(01):169-178.

[4] YU Juan,ZHONG Qinglun,WANG Wenxian,Regional blood supply system optimization under stochastic demand[J].Journal of Computer Applications, 2014,34(09):2585-2589.

[5] LV Xin, Simulation and Optimization Research of Blood Center Inventory Management under Dynamic Demand[J].Industrial Engineering and Management,2011,16(01):118-122 
[6] DUAN Yong-rui,YANG Jin,HUO Jia-zhen,Optimal Ordering and Transfer Policy for Perishable Items with Display Area Inventory Level Dependent Demand[J].Operations Research and Mangement Science, 2014,23(04):51-57. 